\title{
Entretien
}

\section{Temps, histoire et historiographie : entretien avec François Hartog}

\author{
Francine legelski $[*]$
}

* Universidade Federal Fluminense (UFF), Niterói (RJ), Brasil. francineiegelski@hotmail.com

ORCID: https://orcid.org/0000-0001-5026-5048
Résumé : Entretien pour Tempo avec le Professeur François Hartog fait à Paris, le 11 novembre 2019, autour des recherches en historiographie et de son dernier livre, Chronos. L'Occident aux prises avec le temps (sous presse).

Mots-clés : Régime d'historicité ; Coopérations entre le Brésil et la France ; Présentisme ; Historiographie.

Tempo, história e historiografia: entrevista com François Hartog

Resumo: Entrevista concedida pelo professor François Hartog à Tempo em Paris, no dia 11 de novembro de 2019, em que trata de suas investigações em historiografia e de seu mais recente livro, Chronos. L'Occident aux prises avec le temps (no prelo).

Palavras-chave: Regimes de historicidade; Relações BrasilFrança; Presentismo; Historiografia. 


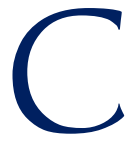

et interview accordé par le professeur François Hartog pour la revue Tempo a eu lieu le 11 novembre 2019 à Paris. ${ }^{1}$ Au cours de l'entretien, le professeur Hartog a parlé de son nouvel ouvrage, Chronos. L'Occident aux prises avec le temps, à paraître en avril 2020. Il a évoqué ses relations avec le Brésil et est revenu, à la lumière du contexte actuel, sur des points importants de sa thèse, élaborée au début des années 2000, sur le régime d'historicité contemporain, qu'il appelle présentisme. Au fil de cet échange, le professeur Hartog nous a également dit où en est l'historiographie aujourd'hui en France.

Francine Iegelski : François Hartog, vous êtes historien, Directeur d'études à l'École des Hautes Études en Sciences Sociales, à Paris, où vous avez mené pendant plus de trente ans vos recherches portant sur l'historiographie ancienne et moderne. Pourriez-vous, pour commencer, nous parler de ce choix de l'histoire ? Qu'est-ce qui l'a motivé et comment, ensuite, regardez-vous aujourd'hui vos années de formation et la relation avec des historiens comme Pierre Vidal-Naquet, auquel vous avez consacré un ouvrage, Vidal-Naquet, historien en personne (2007), Jean-Pierre Vernant, à qui vous avez dédié votre livre Régimes d'historicité (2003), ou encore Michel de Certeau, qui, d'après vous, a été celui qui vous a notamment montré le chemin de l'histoire intellectuelle.

François Hartog : Le choix de l'histoire n'a pas été premier. Quand j'étais à l'École Normale, j'ai hésité entre la philosophie, les lettres et l'histoire et finalement mon choix s'est porté sur l'histoire ancienne. Cela est lié notamment à la lecture faite en ces années-là de quelques livres de Georges Dumézil. Il avait travaillé sur Rome et je me disais, naïvement, que je pourrais faire la même chose sur la Grèce, même s'il disait qu'avec la Grèce, la trifonctionnalité n'était pas opératoire. Il était donc d'autant plus stimulant d'essayer de comprendre pourquoi l'idéologie des trois fonctions n'y fonctionnait pas. Il y avait d'abord cette motivation, cet intérêt intellectuel. L'histoire contemporaine ne m'attirait pas, et la déception de 1968 était encore toute proche. C'est alors que j'ai rencontré Pierre Vidal-Naquet, Jean-Pierre Vernant et Marcel Detienne et leurs façons de questionner la Grèce ancienne me paraissaient neuves et convaincantes. Leur approche, celle d'une anthropologie historique, était critique par rapport à ce qui se faisait alors à la Sorbonne, et elle était en prise avec le mouvement des sciences humaines, avec l'anthropologie structurale de Claude Lévi-Strauss, en particulier. Le choix du monde grec était aussi celui d’un engagement dans les débats intellectuels du moment. Pour

1 Cet entretien a été réalisé au cours de la mission de recherche de l'historienne Francine legelski dans le cadre de l'Accord Capes-Cofecub (Coordenação de Aperfeiçoamento de Pessoal de Nivel Superior/Comitê Francês de Avaliação da Cooperação Universitária com o Brasil) relatif au projet « Idées en temps de guerre froide : circulations intellectuelles, rencontres et rendez-vous manqués», coordonné par les professeurs Denise Rollemberg et Olivier Compagnon. 
eux, les premiers signes de reconnaissance sont venus, en même que s'affirmait et gagnait en visibilité l'Ecole des hautes études en sciences sociales.

FI : Le livre Régimes d'historicité, paru il y a 17 ans, a eu un impact important sur l'historiographie en France et ailleurs, notamment au Brésil. Vous y posez une interrogation d'historien sur le temps. Une interrogation déclenchée par votre diagnostic d'une crise du temps, au cours du dernier tiers du XXe siècle. Considérez-vous aujourd'hui que votre diagnostic, tel qu'avancé et articulé dans le livre [de 2003], concernant les rapports des sociétés contemporaines au temps et la montée du présentisme, est toujours opératoire ? La notion de présentisme est-elle encore un guide pour comprendre les modalités des rapports au temps de notre époque actuelle?

FH : Le diagnostic tel qu'il a été formulé en 2003, était déjà l'aboutissement de plusieurs années d'interrogation qui ont été marquées, pour moi et pour d'autres, par la crise du futur qui s'est manifestée en Europe dès la fin des années 1970 et au début des années 1980. Cette crise du futur s'est traduite, en histoire et plus largement dans l'espace public, par la montée de la mémoire. On commença alors à parler de mémoire, de droit à la mémoire puis bientôt de devoir de mémoire. Tous ces phénomènes qui ont traversé nos sociétés me semblaient, non pas s'expliquer par, mais en tous cas s'inscrire dans cette mise en question de ce qui a été le grand moteur de l'histoire et des sociétés occidentales : le temps moderne, le progrès et la marche vers le progrès. Les facteurs de la mise en question du futur sont multiples, mais un des éléments importants de cette crise a été la montée en puissance de la mémoire de ce qui s'était passé lors de la seconde guerre mondiale, l'extermination des juifs. Elle n'avait certes pas été ignorée dans les années d'après-guerre, mais elle est devenue un thème très important avec le changement des générations. La génération de ceux qui l'avaient directement vécue commençait à disparaître et les générations d'après s'interrogeaient, se posaient des questions, voulaient savoir. C'est dans ce contexte que la thématique de la mémoire s'est peu à peu imposée. Bien d'autres éléments ont joué, particulièrement la crise de 1973, la première grande crise du pétrole qui a frappé les sociétés occidentales. Elles se sont aperçues tout à coup qu'une décision qui frappait le monde entier - l'augmentation du prix du pétrole par les pays producteurs - leur échappait. Un autre facteur évidemment décisif pour expliquer cette crise est l'effritement de l'idée de Révolution qui, jusqu'en 1968, est restée forte. Même si, du côté des pays de l'Est, on ne croyait plus à « l'avenir radieux » du communisme, l'idéal révolutionnaire continuait à être actif à l'Ouest. On sent qu'autour de 1968, au moins en Europe, l'idée perd de sa force : de son évidence. Tous ces éléments, qui relèvent d’une conjoncture générale, ont pour résultat la mise en cause du futur et du temps moderne. Ce qui remplace le futur, c'est le présent, qui, 
certes, a toujours existé mais qui tend à apparaitre désormais comme le seul horizon. C'est pourquoi j'ai proposé de nommer ce phénomène, présentisme, de la même façon qu'a existé le futurisme. Le présentisme est un régime d'historicité dans lequel le présent absorbe le passé et le futur. Ce diagnostic, je le crois toujours valable.

La mémoire et le présentisme ne s'opposent pas. Dans le cas des dictatures en Amérique du Sud, la mémoire est venue après la fin des dictatures avec les demandes de mémoire, la construction de mémoriaux, la justice transitionnelle et les commissions de la vérité. Le présentisme s'est trouvé renforcé au cours des dernières années par les transformations technologiques : le monde numérique est un monde présentiste. Les téléphones portables, les ordinateurs, l'économie et la finance ont pour horizon le présent immédiat. Le capitalisme aujourd'hui est présentiste. Plus que ne l'était le capitalisme industriel. Le capitalisme financier veut des retours sur investissements quasi instantanés. Tout cela maintient, renforce encore le présentisme. Ce que l'on appelle la globalisation est largement fondé là-dessus : elle est fondamentalement présentiste.

La nouveauté aujourd'hui par rapport au début des années 2000 est une mise en question du présentisme, à la suite des insatisfactions qu'il a générées et des injustices dont il est porteur. D'où des tentatives et des souhaits d'en sortir. Il y a toutes sortes de manifestations en ce sens. Et puis, nous le savons, il y a des présentismes, et non pas un présentisme unique : il n'est pas le même pour tous. Il y a les gagnants de la globalisation, les individus les plus flexibles, connectés, mobiles, autant de termes valorisants, qui se donnent aussi comme des impératifs : il faut être rapide, agile, etc. Mais cela ne vaut que pour une frange de la population. À l'autre bout, il y a les nombreux perdants du présentisme, les exclus de ce monde-là, par leur lieu de résidence, leur niveau d'études, leur appartenance sociale et qui se voient, au contraire, réduits à un présentisme de survie : au jour le jour. Il y a des millions de gens dans cette situation, les jeunes précaires qui ne peuvent faire de projets et qui vivent dans un présent subi, ceux qu'on appelle désormais les migrants, les personnes qui vivent très loin des centres urbains. Dans ce cas, la distance spatiale entraîne une distance temporelle, et cette discordance temporelle entraîne aussi des déliaisons sociales dont les mouvements, que l'on voit un peu partout, populistes ou nettement d'extrême droite, font leur fonds de commerce. D’où ces visions si répandues de sociétés qui seraient partagées entre, d'un côté, «l'élite », profitant du «système » et de l'autre, le peuple, le vrai.

FI : Nombre de vos livres sont publiés, lus et font l'objet d'importants débats au Brésil. Vous y êtes déjà allé à différentes reprises et vous connaissez de près les recherches historiques menées au Brésil. Comment s'est opérée cette rencontre avec le Brésil et comment se sont nouées les relations avec les historiens brésiliens ? 
FH : Pour moi, le Brésil a commencé très tôt, bien avant d'y aller ! Avec la lecture du texte de Michel de Certeau sur Jean de Léry; ce fut donc une première rencontre toute livresque. Mais je suis un homme des livres. Quelques années plus tard, ce fut ma rencontre avec Carlos Alberto de Moura Zeron, venu faire son DEA puis sa thèse à l'EHESS, sous ma direction. Thèse sur les jésuites au Brésil. Certeau sert encore une fois de truchement. Sa directrice brésilienne, Janice Theodoro Silva, professeure à l'USP, est entrée en contact avec moi, puis elle m'a invité à Sao Paulo pour y donner des séminaires. Ce fut un premier séjour de quelques semaines. Ensuite, plusieurs doctorants brésiliens sont venus faire leur thèse avec des bourses, à l'époque les bourses étaient généreuses, sur 4 ans, et encore relativement faciles à obtenir, surtout quand il s'agissait de sujets de recherche qui nécessitaient de fréquenter des archives et des bibliothèques en France ou en Europe. Ces étudiants, qui aujourd'hui sont tous professeurs, Zeron, Marcos Veneu, Temístocles Américo Corrêa Cezar, José Otávio Nogueira Guimarães, étaient à l'École des hautes études en sciences sociales qui était un grand centre d'accueil de la colonie étudiante brésilienne, au milieu des années 1980. Il y avait en effet beaucoup de Brésiliens et ceux que j'ai connus étaient partie prenante de cette forte relation avec le Brésil. Il y avait aussi les sociologues, il y avait Alain Touraine et Fernando Henrique Cardoso qui avait été en exil ici. Ce type de lien a donc existé pendant la dictature et s'est poursuivi après. Auparavant encore, il y avait eu Fernand Braudel et, évidemment, Claude Lévi-Strauss à Sao Paulo. Il y a donc une cohorte de personnes qui ont eu des rapports forts avec le Brésil.

La situation a beaucoup changé dans les années 1990, quand les bourses de thèse se sont raréfiées, d'autant que des écoles doctorales s'étaient créées dans les universités brésiliennes. La plupart des professeurs qui y enseignaient avaient été formés en Europe, en France et en Allemagne (pour les historiens) et ils étaient parfaitement à même d'encadrer des cycles doctoraux. La réduction du nombre des bourses était donc légitime et logique. Mais, en termes intellectuels, les «bourses-sandwichs » qui sont apparues surtout dans les années 2000 ne pouvaient pas avoir la même fonction. Quelques mois, ce n'est pas un temps suffisant pour nouer des liens intellectuels durables. De même, pour les post-doctorats : le temps est limité à une année au plus, et le collègue, venu avec un projet précis, est moins disponible intellectuellement qu'un doctorant en pleine formation. Ces transformations ont eu des effets sur l'intensité des liens intellectuels de la recherche en sciences humaines et sociales entre la France et le Brésil. Outre un intérêt pour l'Europe, il y avait aussi du côté des institutions brésiliennes des années 1980 et 1990, le souhait de ne pas tomber sous la dépendance des États-Unis. La puissance des Universités américaines n'était alors pas ce qui intéressait au premier chef les étudiants brésiliens. 
FI : Vous avez dirigé un ouvrage important avec Jacques Revel, Les usages politiques du passé (2001), dans lequel vous abordez, de plusieurs façons, l'instrumentalisation de l'histoire par différents régimes politiques qui souhaitent élaborer un récit du passé à même de servir leurs intérêts politiques. Au Brésil, on assiste aujourd'hui à un affrontement entre certains représentants de la classe politique et des historiens autour du souvenir du passé autoritaire du pays, la dictature militaire (1964-1985). Des hommes politiques de l'actuel gouvernement remettent en question l'historiographie qui traite de cette période et nient aussi bien ce passé autoritaire que les violences et les assassinats perpétrés par l'État Brésilien de l'époque. Que peut l'histoire, que peuvent les historiens de métier devant ces disputes et ces usages, de plus en plus fréquents, du passé à des fins de propagande ?

FH : Premièrement, il n'y a pas lieu d'être surpris car ce n'est pas une démarche inédite, au contraire. En ce qui concerne le Brésil, il y a peut-être un élément qui a joué : au sortir de la dictature, les comptes du régime dictatorial n'ont pas été apurés et le Brésil a vécu sur ce non-assumé. De plus, le système politique brésilien, avec sa Chambre haute (très conservatrice) et les multiples partis avec lesquels il fallait sans cesse composer, n'a probablement pas été en mesure d'affronter ce passé récent. Quand a été créée par Dilma Rousseff la Commission de la vérité, il est déjà bien tard. Le moment où elle aurait pu jouer le rôle d'une justice transitionnelle était probablement passé. Cette initiative a plutôt réveillé l'opposition de certains militaires et, peut-être, je dis bien peut-être, participé à renforcer l'hostilité envers Dilma et à favoriser sa brutale éviction.

Face à une telle conjoncture, que peut l'historien professionnel ? Faire son travail assurément et autant que c'est possible, mais pour ce qui est de peser et de contribuer à ouvrir une réflexion sur le passé, je ne suis pas très optimiste. On le voit en Europe. Il y a, en France par exemple, quelques personnages qui veulent récrire le passé de la France et défendre un roman national soit-disant attaqué ou oublié, tel Éric Zemmour. Il a du succès, indéniablement. Les connaissances historiques que l'on croyait acquises, en fait ne le sont pas. Le passé est toujours susceptible d'être mobilisé, réactivé, instrumentalisé. Prenons un autre exemple, celui de l'Italie : la façon dont Matteo Salvini, le leader populiste de la Ligue du Nord, prétend dire ce qu'est la « véritable » histoire de l'Italie, montre que la période du fascisme mussolinien n'est pas du tout à l'abri de révisions. Ces politiciens sont toujours prêts à réviser l'histoire. Et, le facteur le plus nouveau, que personne ne maîtrise, est la montée en puissance des médias sociaux, avec tous les effets délétères que nous voyons jour après jour un peu partout.

FI : Le dialogue que vous entretenez avec l'anthropologie joue un rôle important dans votre recherche d'historien sur le temps. Dans ce domaine, le nom de Claude Lé- 
vi-Strauss, qui lui aussi a tracé un chemin intellectuel ayant des liens avec le Brésil, a un poids important. Votre article «Le regard éloigné : Lévi-Strauss et l'histoire », publié en 2004 dans la collection L'Herne en hommage à Lévi-Strauss, dirigée par Michel Izard, et qui fut par la suite repris comme un chapitre du livre Évidence de l'histoire (2005), apporte des éléments pour comprendre votre relation avec l'anthropologie structurale. Je pense que votre travail ces dernières décennies a joué un rôle fondamental pour que les historiens en viennent à relire autrement les écrits de Lévi-Strauss sur l'histoire, loin des malentendus et des querelles des années 1960. Pourriez-vous nous parler de votre expérience en tant que lecteur de Lévi-Strauss et de l'importance de ce dernier dans le développement de votre propre réflexion sur les « expériences du temps » ?

FH : Ce que vous dites me fait plaisir mais je n'en suis pas sûr... La réflexion que j'ai menée vous a au moins aidé à formuler vos propres questions. Je m'en réjouis, naturellement, mais ce que j'ai surtout perçu, c'est l'absence de réaction du côté des anthropologues. Pour eux, Lévi-Strauss, c'était fini. Il y a ceux qui ont toujours été contre, les marxistes ou marxisants qui ont toujours dit que Lévi-Strauss, c'était le refus ou la négation de l'histoire. Il y a ceux aussi qui étaient du côté d'un structuralisme pur et dur, qui n'avaient pas d'intérêt pour l'histoire. En France, les Africanistes, qui travaillaient au côté de Georges Balandier, s'intéressaient au présent des sociétés africaines au moment de la décolonisation, estimaient que ces enquêtes menées au milieu des années 1930 autour de toutes petites communautés des hauts-plateaux brésiliens caractérisaient une anthropologie dépassée : primitiviste et dépolitisée. Donc, les anthropologues n'avaient pas envie que l'on leur reparle de Lévi-Strauss, encore moins sous l'angle de son rapport à l'histoire, et les historiens non plus, au moins en France. Pour eux aussi, ces débats n'étaient plus intéressants. Mais, pour moi, il y avait chez Lévi-Strauss la mise en question d'une histoire qui se considérait comme l'Histoire mais qui était, en réalité, une histoire locale, occidentale, européenne, celle de la philosophie de l'histoire de Hegel et de Marx, et encore de Sartre. Il était intéressant de le dire au moment où il l'a dit : à chacun son histoire, au fond. Les débats sur l'idée d'un « hors » de l'histoire, d'un « dans » l'histoire, ou d'un « pas encore » dans l'histoire etc. n'ont plus aucun sens à partir de lui.

Plus directement, à travers sa distinction entre les sociétés chaudes et les sociétés froides (cette différence de température historique) j'ai perçu une suggestion que j'ai traduite par le concept de régime d'historicité. Soutenir que Lévi-Strauss a toujours été attentif à l'histoire et aux modalités du rapport au temps était, en fait, une espèce de provocation. Mais je reste convaincu que cette attention existe chez lui. C'est d'ailleurs ce qui a fait qu'il m'a accompagné dans une grande part de ma vie intellectuelle. A mes 
tout débuts, à la fin des années 1960, il occupait une place importante : la linguistique et l'anthropologie structurale tenaient le haut du pavé. Puis, au fur et à mesure, il est resté présent, à quelque distance, puisque je ne suis pas anthropologue. Il fait même partie de ceux que j'ai nommés les outsiders de l'histoire et que je distingue des insiders: ceux-ci sont des historiens patentés, professionnels, alors que les outsiders sont des savants extérieurs à la discipline mais qui, par leurs interrogations, leurs critiques, leurs jugements sur l'histoire ont joué un rôle important dans les problématiques de celle-ci, même si elle ne le reconnaît pas toujours. Lévi-Strauss a ainsi été la référence, soit explicite, soit implicite, de l'historiographie en France entre 1960 et 1980. Braudel a même pris la peine de distinguer ce qu'était une structure pour les historiens de ce qu'elle était pour un anthropologue. Lévi-Strauss est donc un de ces outsiders qui ont compté : il fait partie d'une longue lignée que je fais commencer avec Aristote et sa Poétique, comme je le rappelais dans la préface à votre thèse que vous m'aviez demandé d'écrire.

FI : En 1993, Lévi-Strauss a écrit un petit texte, «Un autre regard», dans lequel il parle du « réchauffement » des sociétés froides et du « refroidissement » des sociétés chaudes. Vous avez écrit que le présentisme était une espèce d'hyper-réchauffement des sociétés chaudes. Qu'est-ce que vous entendiez par-là?

FH : Le présentisme peut être comparé à une poussée de chaleur. Le temps devient si rapide, si saccadé, si précipité qu'il entre en fusion et tend, à la limite, à disparaitre. Grands consommateurs, d'énergie, les ordinateurs produisent beaucoup de chaleur et, finalement, de l'entropie. Cette entropie ou refroidissement que Lévi-Strauss voyait venir. Comme il l'écrivait déjà à la fin de Tristes tropiques, « un jour l'inertie sera définitive ». Relues aujourd'hui, à la lumière du réchauffement climatique, ces pages pourraient nourrir notre réflexion.

FI : Les travaux de Dipesh Chakrabarty sur l'Anthropocène ont bouleversé la manière dont nous avions l'habitude de comprendre les rapports entre nature et culture, en avançant l'hypothèse selon laquelle nous serions entrés dans une autre époque, nous serions en train de vivre un moment de changement de période historique et géologique. Dans un certain sens, ce serait un nouveau temps, le temps de la Terre, marqué par des questions climatiques. Comment voyez-vous le travail de Chakrabarty par rapport à vos propres recherches sur le présentisme?

FH : Dipesh Chakrabarty, que j'ai eu la chance de rencontrer et avec qui j'ai pu parler longuement lors d'un séjour d'enseignement à Chicago, m'a ouvert le champ, qui se développe avec une rapidité fantastique, des études sur l'Anthropocène. Il est lui-même engagé dans cette réflexion depuis une dizaine d'années, depuis son article séminal sur 
"les 4 thèses sur le climat » (Chakrabarty, 2009). Dans le livre que je viens de finir, Chronos, l'Anthropocène est présent et le surgissement de l'Anthropocène, tout récent (cela fait une dizaine d'années qu'on s'inquiète), signifie évidemment l'introduction du temps très long de la Terre) dans notre paysage. Voilà qui nous désarçonne d'autant plus que nous étions plus ou moins installés, bien ou mal, dans le présentisme, dans lequel le long futur du régime moderne d'historicité n'avait plus cours. Le seul futur était un futur immédiat. Et tout à coup, surgit un futur incroyablement long, mais sur lequel on n'a pas prise.

L'hypothèse que je développe dans Chronos est que le temps moderne s'était débarrassé des limites qui bornaient le temps chrétien. Le régime chrétien d'historicité est, en effet, pris entre l'Incarnation et la Parousie, entre l'Incarnation et l'Apocalypse. Pour les chrétiens, dès les premiers temps du christianisme, l'Incarnation ouvre un temps complètement nouveau qui est aussi le début du temps de la fin. Ce sera le grand thème, puissamment orchestré par Saint Augustin, de la vieillesse du monde. S'ouvre un temps de la fin, mais qui n'est pas (encore) la fin des temps. La fin, elle, n'est pas à la disposition des hommes. Seul Dieu en décide. Ce sera le message constamment repris de l'Église catholique. Oui, le temps de la fin approche mais nul ne sait quand la fin, elle-même, interviendra. Cette structure singulière, constitutive du temps occidental, avec ce binôme «temps de la fin / fin des temps » est justement ce que les modernes font disparaître, quand il se mettent à explorer l'âge de la Terre. Du côté de la géologie et du passé, le grand repère est Buffon, dans la direction du futur, c'est Condorcet, ouvrant la perspective d'un avenir «indéfini ». Depuis la fin du XVIIIe siècle, le monde moderne a vécu sur cette vision du temps. A cette échelle, le présentisme n'est guère plus qu'une bulle, à l'intérieur de laquelle le passé et le futur n'ont guère de place.

Mais avec l'Anthropocène, se trouvent simultanément réintroduits un passé très long et un futur, plus long encore. Dans la mesure où l'Anthropocène est porteur d'une catastrophe possible, est réintroduite du même coup l'idée d'une limite, d'une fin : non pas de la Terre ni du temps de la Terre, mais du temps des hommes, du temps du monde, du temps historique. Et dès lors que vous posez la fin, une fin au moins possible, vous introduisez également l'idée d'un temps de la fin. Si la fin approche, cela veut dire que nous sommes entrés dans le temps de la fin. D'où, tous ces gens qui, à des titres divers, croient urgent de nous communiquer les résultats de leurs calculs de la date de la fin, de l'effondrement, de la catastrophe, de l'apocalypse. Les appellations varient. Mais se réinstalle un cadre de pensée dans lequel le monde occidental a vécu pendant au moins dix-huit siècles, qui est scandé par le binôme fin des temps / temps de la fin. C'est cela que je me suis efforcé d'explorer dans Chronos. Au fond, ce que je tire des études sur l'Anthropocène (je ne peux rien dire sur l'Anthropocène en tant que tel, n'étant ni géo- 
logue, ni climatologue, ni même historien des sciences), c'est la possibilité d'élargir ma réflexion sur les rapports au temps et sur les usages du concept de régime d'historicité.

FI : Cette question du temps chrétien dont vous traitez dans votre livre Chronos fait depuis longtemps partie de vos recherches. Il y a donc longtemps que vous pensiez à cela... FH : Oui, un certain nombre d'années. Je ne savais pas encore de quelle manière mais oui, au fond, le temps chrétien et le régime chrétien d'historicité étaient déjà présents dans le livre Régimes d'historicité mais n'étaient pas du tout développés. Je me suis sérieusement posé la question : existe-t-il un régime chrétien d'historicité et de quoi est-il donc fait ? Je suis reparti des Apocalypses juives et premiers textes du christianisme, pour arriver à cette définition : le régime chrétien d'historicité, est un présentisme apocalyptique.

FI : Vos livres sont truffés de références à l'art et plus particulièrement à la littérature, comme des traces qui vous permettent de poser autrement la question des rapports qu'une société entretient avec le temps. Je me souviens du lien que vous avez proposé entre une sculpture d'Anselm Kiefer, L'Ange de l'histoire, et le présentisme (Hartog, 2013, p.155-161). Pourriez-vous nous en dire un peu plus sur votre vision de cette relation entre art et histoire?

FH : Plus à la littérature qu'à la peinture mais oui, à la peinture aussi. J'ai toujours été hostile à lídée d'un usage instrumental de la littérature, comme si la littérature devait fournir des documents pour l'histoire, tout exprès à l'usage des historiens. Les débats interminables sur l'histoire et la littérature qui reviennent périodiquement (on l'a vu récemment encore), ne sont pas très intéressants. Il y a quelque chose que la littérature fait et que l'historien, ou tout praticien des sciences sociales, ne peut pas faire. En effet, le sociologue, le géographe, le philosophe, tout comme l'historien, arrivent après la bataille. Il faut à l'historien un certain recul pour dire ce qui s'est passé. Il est forcément dans une démarche réflexive. C'est au crépuscule que se lève la chouette de Minerve, disait Hegel. L'écrivain, non. Ce qui fait la grandeur et la singularité de l'écrivain, c'est vrai aussi du peintre ou du musicien, c'est qu'il cherche à dire ce qui n'a encore jamais été dit, peint ou composé. Autrement dit, il n'a pas besoin de ce temps (si bref qu'il soit), de ce recul, de cet écart dont le praticien des sciences sociales ne peut se passer. Ce qui permet à l'écrivain de saisir quelque chose de ce qui est en train de se passer et qui n'a pas encore les mots pour se dire. Le fait de chercher à dire ce qui n'a pas été dit lui donne une liberté plus grande qu'à celui qui est soumis à des règles, doit apporter des preuves, des références, afin de permettre à d'autres de refaire le même chemin. L'écrivain n'a nul besoin de s'embarrasser de tout cet appareillage, mais évidemment il prend plus de 
risques puisqu'il peut manquer la chose, se tromper, s'égarer. Ou, s'il renonce à essayer de dire ce qui n'a jamais été dit, il peut devenir celui qui redit ce qui a été dit, et une bonne part de la littérature ou de la peinture fait ça... C'est cette singularité de la position de l'artiste que j'essaie de saisir. Il précède le chercheur, nécessairement. Ce n'est pas pour autant une vision romantique de l'artiste en mage ou en prophète que je suggère!

FI : Vos travaux et les nombreuses interrogations que vous avez évoquées témoignent de l'importance acquise par les études d'historiographie au sein des recherches historiques (Hartog, 1990-1991, p.128-129). Pensez-vous que le champ de l'historiographie, qui a fait l'objet pendant longtemps de la méfiance des historiens, associé à la philosophie de l'histoire, a finalement acquis droit de cité ? Quelle est la situation faite à l'historiographie dans le paysage universitaire français ? Où en sont les travaux d'historiographie en France, ou en Europe, aujourd'hui ? Que peut-on attendre des jeunes recherches?

FH : Quand j'ai commencé à travailler, on entrait dans un moment historiographique, en prenant l'expression en plusieurs sens. C'était justement le moment où, de divers côtés, la démarche réflexive était valorisée. Cela coïncidait avec les premières mises en question du futur. De fait, des institutions, des disciplines, des entreprises, des gens ordinaires s'interrogeaient sur leur passé. Après l'accélération de la période qu'on a appelé les Trente Glorieuses (1945-1975), le besoin de faire le point, de faire une pause, de regarder en arrière, se faisait sentir. C'est un moment archivistique et généalogique, au sens propre. Les services d'archives ont alors connu un afflux considérable de gens se mettant à la généalogie. Un deuxième sens du terme historiographie est celui, bien établi, d'histoire de l'histoire : une histoire de la discipline et de ses praticiens, telle que souhaitait la promouvoir Charles-Olivier Carbonell dans les années 1980. Un troisième sens, enfin, est l'historio-graphie, au sens d'une attention portée à l'écriture de l'histoire, à l'histoire comme pratique d'écriture. Ce questionnement a eu comme repère majeur, en France, L'écriture de l'histoire (1975) de Michel de Certeau. En cette acception, l'historiographie est liée à la place alors occupée par la linguistique et la sémiologie (le récit, le discours, l'énonciation). Pour moi, il était entendu que j'étais alors plus intéressé par la question de l'écriture de l'histoire, dont mon livre sur Hérodote est la traduction, mais sans négliger, tant s'en faut, l'importance de l'œuvre d'Arnaldo Momigliano, dont l'érudition historiographique (au deuxième sens du terme) était considérable.

Mais l'attention portée à la dimension d'écriture de l'histoire a été fortement mise en question dans les années suivantes, lorsque s'est développé, d'abord aux Etats-Unis, ce qui s'est appelé le Linguistic turn. En histoire, le débat a vite dérivé vers des simplifications outrancières. Si tout est langage, alors le réel disparaît, et il n'y a plus de différence 
entre l'histoire et la fiction. Le champion ou le vilain du Linguistic turn a été Hayden White et face à lui s'est dressé Carlo Ginzburg. Contre ceux qu'il nommait les sceptiques, il défendait le réel et la vérité de l'histoire. Le point fort de la polémique a tourné autour du négationnisme. Les négationnistes, qui se nommaient révisionnistes, soutenaient que les chambres à gaz n'avaient pas existé, prétendant qu'aucune preuve de leur existence n'avait été apportée et qu'on ne pouvait produire aucun témoin. Pierre Vidal-Naquet les a justement désignés comme « les assassins de la mémoire ». Or, si l'histoire n'est que discours, si le « fait n'a jamais qu'une existence linguistique » (Barthes), alors on peut aussi bien arriver à soutenir que les chambres à gaz ont existé ou n'ont pas existé. Je ne reprends pas ici ce que j'ai écrit sur cette polémique dans mon livre, Croire en l'histoire. Le lecteur intéressé peut s'y reporter. Résumer l'argumentation en quelques phrases est, en effet, difficile. Je retiens ce seul point : le danger de glisser (même sans l'avoir voulu) d'interrogations sur l'écriture de l'histoire vers des positions possiblement négationnistes mit fin au débat chez les historiens. On laissa Paul Ricoeur officier dans Temps et récit comme juge de paix. Il indiquait ce qu'on pouvait garder chez Hayden White et là où il allait trop loin. Il suffirait désormais de renvoyer à Ricoeur (1983).

Aujourd'hui, on est dans un moment, me semble-t-il, où la dimension réflexive n'intéresse plus guère. On veut de l'émotion et du sensible. On parle surtout de «terrain », d'enquêtes de terrain, d'archives et d'identités. "Théorie » est devenue un gros mot. Jusqu'où ira cette tendance, et combien de temps elle durera, je l'ignore? Dans cette conjoncture, il est clair que l'historiographie (à tous les sens du terme) attire nettement moins : de quel enjeu pourrait-elle être encore porteuse? A l'École des Hautes Études en Sciences Sociales, depuis que j'ai pris ma retraite, il n'y a plus de chaire d'historiographie. C'est à tout le moins un indice.

FI : Que pourriez-vous dire en faveur de l'historiographie?

FH : Je continue à penser que la démarche réflexive est absolument indispensable. On peut la mener de différentes façons. L'historiographie peut s'entendre comme participant d'une histoire conceptuelle, d'une histoire intellectuelle, d'une histoire des disciplines; elle n'a pas à craindre de se revendiquer aussi comme philosophie de l'histoire. Au cours de ma longue enquête sur les formes changeantes du rapport au temps que les sociétés ont entretenues au cours des siècles, je me suis efforcé de la pratiquer de la manière la plus ouverte qui soit, traversant les siècles, les œuvres, les milieux et les lieux, sans jamais perdre de vue le présent énigmatique : indéductible, disait Paul Valéry. 


\section{Références}

BRAUDEL, Fernand. Histoire et sciences sociales: la longue durée. Annales. Histoire, Sciences Sociales (Paris). v. 13, n. 4, p. 725-753, 1958.

CERTEAU, Michel de. L'écriture de l'histoire. Paris: Gallimard, 1975. (Bibliothèque des Histoires)

CHAKRABARTY, Dipesh. The climate of history: four theses. Critical Inquiry (Chicago). v. 35, n. 2, p. 197-222, 2009.

HARTOG, François. Historiographie, In: Annuaire de l'École des Hautes Etudes en Sciences Sociales. Comptes rendus des cours et conférences. p. 128-129, 1990-1991.

HARTOG, François. Régimes d'historicité: présentisme et expériences du temps. Paris: Éditions du Seuil, 2003.

HARTOG, François. Le regard éloigné: Lévi-Strauss et l'histoire. In: Izard, Michel (Dir.). Claude
Lévi-Strauss. Paris: Éditions de l'Herne, p. 313-319, 2004.

HARTOG, François. Évidence de l'histoire: ce que voient les historiens. Paris: Éditions de l'École des Hautes Etudes en Sciences Sociales, 2005.

HARTOG, François. Vidal-Naquet, historien en personne: l'homme-mémoire et le moment-mémoire. Paris: La Découverte, 2007.

HARTOG, François. Croire en l'histoire. Paris: Flammarion, 2013.

HARTOG, François; REVEL, Jacques (Dir.). Les usages politiques du passé. Paris: Éditions de l'École des Hautes Etudes en Sciences Sociales, 2001.

LÉVI-STRAUSS, Claude. Un autre regard, L'Homme (Paris). v. 33, n. 126, p. 7-11, 1993.

RICOEUR, Paul. Tempset récit. Tome 1. Paris: Seuil, 1983. 